Chronic Obstructive Pulmonary Diseases:

Journal of the COPD Foundation

\author{
Brief Communication
}

\title{
Maintaining Emotional Well-Being During the COVID-19 Pandemic: A Resource for Your Patients
}

Sara Latham, $\mathrm{BA}^{1}$ Jamie Sullivan, $\mathrm{MPH}^{1}$ Stephanie Williams, $\mathrm{RRT}^{1}$ Michelle N. Eakin, $\mathrm{PhD}^{2}$

Citation: Latham S, Sullivan J, Williams S, Eakin MN. Maintaining emotional well-being during the COVID-19 pandemic: a resource for your patients. Chronic Obstr Pulm Dis. 2020;7(2):76-78. doi: https://doi.org/10.15326/jcopdf.7.2.2020.0150

1 COPD Foundation, Washington, DC

2 Division of Pulmonary and Critical Care Medicine, Johns Hopkins University, Baltimore, Maryland

\section{Address correspondence to:}

Michelle N. Eakin, PhD

Johns Hopkins University

5501 Hopkins Bayview Circle Room 4b.74

Baltimore, MD 21224

Email: Meakin1@jhmi.edu

\section{Introduction}

Throughout this current COVID-19 pandemic, the COPD Foundation remains committed to providing our respiratory community with accurate, timely information. While we do not attempt to replace a physician's medical advice or provide information that is contradictory to the COVID-19 guidelines of the Centers for Disease Control and Prevention ${ }^{1}$ and the World Health Organization, ${ }^{2}$ the Foundation has attempted to answer the many COVID-19 questions received from the high-risk populations we represent (bronchiectasis, nontuberculous mycobacteria and chronic obstructive pulmonary disease) and provide updated medical information about managing these diseases during this public health crisis through a static COVID-19 web page, ${ }^{3}$ blog posts ${ }^{4}$ and webinars. ${ }^{3}$

As we monitor inquiries, concerns and conversations from our COPD community via the Foundation's robust $C O P D 360$ social interactive site, ${ }^{5}$ we realize our COPD community has a unique burden. As an identified "COVID-19 high-risk population" our COPD community must not only vigilantly adhere to all public health guidelines for avoiding and stopping the spread of COVID-19, but they must also maintain their own, often-fragile lung health, monitoring symptoms for potential COPD exacerbations, which can often mimic and be confused with COVID-19 symptoms. This unique burden can be a source of heightened stress and anxiety.

The current COVID-19 pandemic is impacting every aspect of daily life and is emotionally challenging for us all. Stress and anxiety are increasing for everyone as we attempt to adjust our daily activities to adhere to social distancing which is impacting work, social, educational, leisure and entertainment activities, while we are also troubled with increased worries about personal health, the health of loved ones and the overall health of our communities.

Yet for individuals with COPD the situation is intensified as these individuals are already at an increased risk for anxiety and depression-two common COPD comorbidities. In fact, individuals with COPD are at a higher risk of having anxiety (median prevalence 16\%, range 10\%-55\%) and depression (median prevalence 17\%, range 0\%-42\%) than individuals with other chronic conditions. ${ }^{6}$

In addition, the awareness of being a part of an identified, "COVID-19 high risk population" is a unique source of anxiety, depression and stress for individuals with COPD. This can also be further exacerbated by social isolation, difficulty accessing appropriate medical care and increased financial strain.

In our continued attempt to provide our COPD community with resources to mitigate this on-going, global respiratory emergency, we offer a patientfocused informational resource "Maintaining Emotional Well-Being: A Resource for COPD Patients During the COVID-19 Pandemic". 
We encourage you to share this link with your patients and their caregivers and families. This informational pdf is downloadable, printable and easily shared via this link: https://www.copdfoundation.org/downloads/ MainEmtn-Health-FINAL.pdf 


\section{References}

1. Centers for Disease Control and Prevention. People who are at higher risk for severe illness. Updated April 15, 2020. Accessed April 17, 2020. https://www.cdc.gov/coronavirus/2019-ncov/ need-extra-precautions/groups-at-higher-risk.html

2. World Health Organization. Coronavirus disease (COVID-19) advice for the public. Published March 31, 2020. Accessed April 17, 2020. https://www.who.int/emergencies/diseases/ novel-coronavirus-2019/advice-for-public

3. COPD Foundation. Coronavirus information for the COPD community. Updated April 16, 2020. Accessed April 17, 2020. https://www.copdfoundation.org/Learn-More/I-am-a-Personwith-COPD/Coronavirus-Information.aspx

4. COPD Foundation. A coronavirus update for the COPD community-blog posts. Updated April 8, 2020. Accessed April 17, 2020. https://www.copdfoundation.org/COPD360social/ Community/COPD-Digest/Article/1553/A-CoronavirusUpdate-for-the-COPD-Community.aspx

5. COPD Foundation. COPD360social. Activity feed. Accessed April 17, 2020. https://www.copdfoundation.org/ COPD360social/Community/Activity-Feed.aspx

6. Willgoss TG. Yohannes AB. Anxiety disorders in patients with COPD: a systematic review. Respir Care. 2013; 58(5): 858-866. doi: https://doi.org/10.4187/respcare.01862 